\title{
Nonlinear perturbations of linear evolution systems*
}

\author{
By Robert H. MARTIN, Jr.
}

(Received Nov. 5, 1975)

(Revised June 21, 1976)

It is the purpose of this paper to study the existence and behavior of "mild" solutions to abstract differential equations in Banach spaces. Roughly speaking, the "right hand side" of these equations are assumed to be the sum of two operators, one being the generator of a linear (or affine) evolution system and the other being continuous and nonlinear. The results and techniques established here are improvements of those given by the author in [2].

In $\$ 1$ we indicate the basic notations, and the fundamental results on the existence of approximate solutions are developed in $\$ 2$. Criteria for local existence of solutions is given in $\S 3$, and $\S 4$ is devoted to establishing a relationship of these ideas to inequalities. An example illustrating the abstract results is studied in $\$ 5$.

\section{$\S 1$. Preliminaries.}

Let $X$ be a Banach space over the real or complex field and let $|\cdot|$ denote the norm on $X$. Also, let $-\infty<a<b \leqq+\infty$ and let $\Delta=\{(t, s): a \leqq s \leqq t<b\}$. Throughout this paper $T=\{T(t, s):(t, s) \in \Delta\}$ is a family of bounded linear operators on $X$ which satisfy

$$
T(t, t)=I \text { and } T(t, s) T(s, r)=T(t, r) \text { for } a \leqq r \leqq s \leqq t<b ;
$$

and

(T2) the map $(t, s) \rightarrow T(t, s) x$ is continuous from $\Delta$ into $X$ for each $x \in X$.

From (T2) and the principle of uniform boundedness we also have that

(T3) for each $c \in[a, b)$ there is a number $M_{c} \geqq 1$ such that

$$
\|T(t, s)\| \equiv \sup \{|T(t, s) x|:|x| \leqq 1\} \leqq M_{c} \text { for all } a \leqq s \leqq t \leqq c .
$$

Now suppose that $\alpha:[a, b) \rightarrow X$ is continuously differentiable, $\beta:[a, b) \rightarrow X$ is continuous, and define the family $S=\{S(t, s):(t, s) \in \Delta\}$ of (affine) mappings on $X$ by

* This work was supported by the U.S. Army Research Office, Durham, North Carolina. 


$$
\begin{gathered}
S(t, s) x=T(t, s)(x-\alpha(s))+\int_{s}^{t} T(t, r) \beta(r) d r+\alpha(t) \\
\text { for } a \leqq s \leqq t<b \text { and } x \in X .
\end{gathered}
$$

One may easily check that $S(t, t)=I, S(t, s) S(s, r)=S(t, r)$ for $a \leqq r \leqq s \leqq t<b$, and for each $x \in X,(t, s) \rightarrow S(t, s) x$ is continuous from $\Delta$ into $X$. From (T3) it also follows that

$$
|S(t, s) x-S(t, s) y| \leqq M_{c}|x-y| \quad \text { for } x, y \in X, a \leqq s \leqq t \leqq c .
$$

In particular, it is easy to see that

$$
(t, s, x) \rightarrow S(t, s) x \text { is continuous from } \Delta \times X \text { into } X .
$$

Let $D$ be a subset of $[a, b) \times X$ with the following two properties: $D$ is locally closed (i.e., for each $(t, z) \in D$ there is an $R>0$ such that $\{(s, x) \in D$ : $|t-s|,|z-x| \leqq R\}$ is closed in $\boldsymbol{R} \times X)$ and for each $t \in[a, b)$ the set $D(t) \equiv$ $\{x \in X:(t, x) \in D\}$ is nonempty. Finally, suppose that

$$
D(B) \subset[a, b) \times X, D \subset D(B) \text { and } B: D(B) \rightarrow X \text { is continuous. }
$$

The purpose of this paper is to study the existence of solutions to the integral equation

$$
u(t)=S(t, a) z+\int_{a}^{t} T(t, r) B(r, u(r)) d r
$$

where $z \in D(a)$ and $u$ is a continuous function from some subinterval $J$ of $[a, b)$ (with $a \in J$ ) into $X$ such that $u(t) \in D(t)$ for all $t \in J$. The linear family $T$ is said to be generated if for each $t \in[a, b)$ there is a densely defined linear operator $L(t)$ on $X$ such that

$$
L(t) x=\lim _{h \rightarrow 0+}[T(t+h, t) x-x] / h \quad \text { for all } x \in D(L(t)),
$$

where $D(L(t))$ is the set of all $x \in X$ such that this limit exists. The family of operators $L=\{L(t): t \in[a, b)\}$ is called the generator of $T$. If $T$ is generated with generator $L$ and $t \in[a, b)$, define

$$
A(t) x=L(t)(x-\alpha(t))+\beta(t)+\alpha^{\prime}(t) \quad \text { for all } x \in D(A(t))
$$

where $D(A(t))=\{x \in X: x-\alpha(t) \in D(L(t))\}$. It is routine to check that

$$
A(t) x=\lim _{h \rightarrow 0+}[S(t+h, t) x-x] / h \text { for all } t \in[a, b) \text { and } x \in D(A(t)) .
$$

In particular, if a solution $u$ to (1.4) is differentiable on $J$, then $u(t) \in D(A(t))$ for all $t \in J$ and

$$
u^{\prime}(t)=A(t) u(t)+B(t, u(t)) \quad \text { for } t \in J \text { and } u(a)=z .
$$

Therefore, differentiable solutions to (1.4) are solutions to (1.7). However, in 
this paper we consider only the existence of solutions to (1.4).

\section{§ 2. Approximate solutions.}

In this section we retain the notations of $\S 1$. If $\varepsilon>0$ and $\rho \in(a, b)$, a function $\phi:[a, \rho] \rightarrow X$ is an $\varepsilon$-approximate solution to (1.4) on $[a, \rho]$ if the following conditions hold: there is a partition $\left\{t_{i}\right\}_{0}^{N}$ of $[a, \rho]$ with $t_{i}-t_{i-1} \leqq \varepsilon$ for $i=1, \cdots, N$ such that

$$
\begin{aligned}
& \phi(a)=z \text { and } \phi\left(t_{i}\right) \in D\left(t_{i}\right) \text { for } i=1, \cdots, N ; \\
& \phi(t)=S\left(t, t_{i}\right) \phi\left(t_{i}\right)+\int_{t_{i}}^{t} T(t, r) B\left(t_{i}, \phi\left(t_{i}\right)\right) d r
\end{aligned}
$$

$$
\text { for } t \in\left[t_{i}, t_{i+1}\right) \text { and } i=0, \cdots, N-1 \text {; }
$$

and

(ع3) $\left|\phi\left(t_{i+1}-\right)-\phi\left(t_{i+1}\right)\right| \leqq \varepsilon\left(t_{i+1}-t_{i}\right)$ for $i=0, \cdots, N-1$.

We associate with $\phi$ a function $\gamma\left(=\gamma_{\dot{\phi}}\right)$ from $[a, \rho]$ into $[a, \rho]$ defined by

$$
\gamma(\rho)=\rho \text { and } \gamma(t)=t_{i} \text { whenever } t \in\left[t_{i}, t_{i+1}\right) \text {. }
$$

Moreover, for each $i \in\{0, \cdots, N-1\}$ define the function $\phi\left(\cdot, t_{i}\right)\left(=\phi_{\phi}\left(\cdot, t_{i}\right)\right)$ from $\left[t_{i}, \rho\right]$ into $X$ by

$$
\phi\left(t, t_{i}\right)=S\left(t, t_{i}\right) \phi\left(t_{i}\right)+\int_{t_{i}}^{t} T(t, r) B(\gamma(r), \phi(\gamma(r))) d r .
$$

One should note that $\phi\left(\cdot, t_{i}\right)$ is continuous on $\left[t_{i}, \rho\right]$.

Lemma 2.1. Suppose that $\phi$ and $\phi$ are as above. Then

$$
\left|\phi(t)-\phi\left(t, t_{i}\right)\right| \leqq M_{\rho}\left(t_{j-1}-t_{i}\right) \varepsilon
$$

for each $0 \leqq i<j \leqq n$ and $t \in\left[t_{j-1}, t_{j}\right)$.

Proof. The proof is by induction on $j-i$. It is immediate from $(\varepsilon 2)$ and (2.2) that (2.3) holds for $j-i=1$, so suppose that (2.3) holds for $j-i=k$ where $1 \leqq k<n-1$, and let $j-i=k+1$. Noting that

$$
S(t, r) x+T(t, s) y=S(t, s)[S(s, r) x+y]
$$

for $x, y \in X$ and $a \leqq r \leqq s \leqq t<b$, it follows that

$$
\begin{aligned}
& \left|\phi\left(t, t_{i}\right)-\phi\left(t, t_{i+1}\right)\right| \\
& \left.=\mid S\left(t, t_{i}\right) \phi\left(t_{i}\right)+T\left(t, t_{i+1}\right) \int_{t_{1}}^{t i+1} T\left(t_{i+1}, r\right) B\left(t_{i}, \phi\left(t_{i}\right)\right)\right) d r-S\left(t, t_{i+1}\right) \phi\left(t_{i+1}\right) \mid \\
& =\left|S\left(t, t_{i+1}\right) \phi\left(t_{i+1}-\right)-S\left(t, t_{i+1}\right) \phi\left(t_{i+1}\right)\right| \\
& \leqq M_{\rho}\left|\phi\left(t_{i+1}-\right)-\phi\left(t_{i+1}\right)\right| \leqq M_{\rho}\left(t_{i+1}-t_{i}\right) \varepsilon
\end{aligned}
$$

for all $t_{i+1} \leqq t \leqq \rho$. Therefore, if $j=i+k+1$ and $t \in\left[t_{j-1}, t_{j}\right)$, then $j-(i+1)=k$ 
and by the induction hypothesis it follows that

$$
\begin{aligned}
\left|\phi(t)-\phi\left(t, t_{i}\right)\right| & \leqq\left|\phi(t)-\psi\left(t, t_{i+1}\right)\right|+\left|\phi\left(t, t_{i+1}\right)-\phi\left(t, t_{i}\right)\right| \\
& \leqq M_{\rho}\left(t_{j-1}-t_{i+1}\right) \varepsilon+M_{\rho}\left(t_{i+1}-t_{i}\right) \varepsilon \\
& =M_{\rho}\left(t_{j-1}-t_{i}\right) \varepsilon .
\end{aligned}
$$

This completes the proof.

Our aim now is to obtain criteria which assures the existence of approximate solutions to (1.4). Let $z \in D(a)$ and using the continuity of $T, S$ and $B$ as well as the fact that $D$ is locally closed, choose positive numbers $R, \varepsilon_{0}$, $\sigma$ and $P$ so that the following conditions are fulfilled:

(C1) $F \equiv\{(t, x) \in D:|t-a|,|x-z| \leqq R\}$ is closed in $\boldsymbol{R} \times X$ and

$|B(t, x)| \leqq P$ for all $(t, x) \in F$;

(C2) $a<\sigma<b, \sigma+\varepsilon_{0}<b$ and $\sigma+\varepsilon_{0}-a<R$;

(C3) if $M \equiv M_{\sigma+\varepsilon_{0}}$ then $|S(t, a) z+y-z| \leqq R / 2$ whenever $t \in\left[a, \sigma+\varepsilon_{0}\right]$ and $y \in X$ with $|y| \leqq\left(\sigma+\varepsilon_{0}-a\right) M \cdot P$;

and

(C4) $\quad\left(\sigma+\varepsilon_{0}-a\right) M \varepsilon_{0} \leqq R / 2$.

LEMmA 2.2. Suppose that (C1)-(C4) hold, $\rho \in\left(a, \sigma+\varepsilon_{0}\right], \varepsilon \leqq \varepsilon_{0}$, and $\phi$ is an $\varepsilon$-approximate solution to (1.4) on $[a, \rho]$. Then $\left(t_{i}, \phi\left(t_{i}\right)\right) \in F$ for each $i=0, \cdots, N$.

Proof. Let $\psi$ be defined by (2.2). The proof is by induction on $i$. Since $\phi\left(t_{0}\right)=z$, assume that $\left(t_{i}, \phi\left(t_{i}\right)\right) \in F$ for $i=0, \cdots, k$ where $0 \leqq k<n$. Since

$$
\left|\int_{a}^{t} T(t, r) B(\gamma(r), \phi(\gamma(r))) d r\right| \leqq \int_{a}^{t} M \cdot P d r \leqq(t-a) M \cdot P
$$

for all $t \in\left[t_{k}, t_{k+1}\right)$, it follows from (C3) that $|\psi(t, a)-z| \leqq R / 2$ for $t \in\left[t_{k}, t_{k+1}\right)$. Hence, from Lemma 2.1, (C4) and ( $\varepsilon 3$ ),

$$
\begin{aligned}
& \left|\phi\left(t_{k+1}\right)-z\right| \leqq\left|\phi\left(t_{k+1}\right)-\phi\left(t_{k+1}-\right)\right|+\lim _{t_{t \rightarrow t^{-}}}|\phi(t)-z| \\
& \quad \leqq\left(t_{k+1}-t_{k}\right) \varepsilon+\lim _{t \rightarrow t_{k+1^{-}}}|\phi(t)-\phi(t, a)|+R / 2 \\
& \quad \leqq M\left(t_{k+1}-t_{k}\right) \varepsilon+M\left(t_{k}-a\right) \varepsilon+R / 2 \leqq R
\end{aligned}
$$

which establishes the assertion by induction.

For each $x \in X$ and $E \subset X$ define $d(x ; E)=\inf \{|x-y|: y \in E\}$. Our crucial assumption for the existence of approximate solutions is

$$
\liminf _{h \rightarrow 0+} d(S(t+h, t) x+h B(t, x) ; D(t+h)) / h=0 \text { for all }(t, x) \in F \text {. }
$$

Noting that 


$$
\int_{t}^{t+h} T(t+h, r) B(t, x) d r-h B(t, x)=o(h)
$$

where $h^{-1}|o(h)| \rightarrow 0$ as $h \rightarrow 0+$, one sees that (C5) is equivalent to the condition

$$
\begin{gathered}
\liminf _{h \rightarrow 0+} d\left(S(t+h, t) x+\int_{t}^{t+h} T(t+h, r) B(t, x) d r ; D(t+h)\right) / h=0 \\
\text { for all }(t, x) \in F .
\end{gathered}
$$

Under conditions (C1)-(C5) we have the following basic result on the existence of approximate solutions to (1.4):

Proposition 2.1. Suppose that (C1)-(C5) are fulfilled and $0<\varepsilon \leqq \varepsilon_{0}$. Then there is a $\rho \in\left[\sigma, \sigma+\varepsilon_{0}\right)$ such that (1.4) has an $\varepsilon$-approximate solution $\phi$ on $[0, \rho]$. Moreover, in addition to $(\varepsilon 1)-(\varepsilon 3), \phi$ also satisfies

$$
\left|S\left(t, t_{i}\right) \phi\left(t_{i}\right)-\phi\left(t_{i}\right)\right| \leqq \varepsilon \text { for } t \in\left[t_{i}, t_{i+1}\right), i=0, \cdots, N-1 ;
$$

and

$$
\begin{aligned}
& \text { if }(t, y) \in D(B) \text { with } t \in\left[t_{i}, t_{i+1}\right] \text { and } \\
& \left|y-\phi\left(t_{i}\right)\right| \leqq\left(t_{i+1}-t_{i}\right) P+\max \left\{\left|\left[S\left(t_{i}+h, t_{i}\right)-I\right] \phi\left(t_{i}\right)\right|: 0 \leqq h \leqq t_{i+1}-t_{i}\right\}
\end{aligned}
$$

then

$$
\left|B(t, y)-B\left(t_{i}, \phi\left(t_{i}\right)\right)\right| \leqq \varepsilon .
$$

PROOF. The proof techniques follow Webb [4] and Martin [2], and both $\phi$ and the partition $\left\{t_{i}\right\}_{0}^{N}$ are constructed by induction. So set $t_{0}=a, \phi\left(t_{0}\right)=z$, and assume that $i$ is a nonnegative integer and $\phi$ is constructed on $\left[a, t_{i}\right]$ where $a \leqq t_{i}<\sigma+\varepsilon_{0}$. If $t_{i} \geqq \sigma$ set $\rho=t_{i}$ and if $t_{i}<\sigma$ choose a number $\delta_{i}$ as follows :

$$
\delta_{i} \in[0, \varepsilon] \text { and } t_{i}+\delta_{i} \leqq \sigma+\varepsilon_{0} ;
$$

(2) if $(t, y) \in D(B)$ with $t \in\left[t_{i}, t_{i}+\delta_{i}\right]$ and

$$
\begin{aligned}
& \left|y-\phi\left(t_{i}\right)\right| \leqq \delta_{i} P+\max \left\{\left|\left[S\left(t_{i}+h, t_{i}\right)-I\right] \phi\left(t_{i}\right)\right|: 0 \leqq h \leqq \delta_{i}\right\} \text { then } \\
& \mid B(t, y)-B\left(t_{i}, \phi\left(t_{i}\right)|,|\left[S\left(t, t_{i}\right)-I\right] \phi\left(t_{i}\right) \mid \leqq \varepsilon .\right.
\end{aligned}
$$

$$
d\left(S\left(t_{i}+\delta_{i}, t_{i}\right) \phi\left(t_{i}\right)+\int_{t_{i}}^{t_{i}+\delta_{i}} T\left(t_{i}+\delta_{i}, r\right) B\left(t_{i}, \phi\left(t_{i}\right)\right) d r ; D\left(t_{i}+\delta_{i}\right)\right) \leqq \delta_{i} \varepsilon / 2 ;
$$

(4) if (1)-(3) hold with $\delta_{i}$ replaced by $\eta$, then $\delta_{i} \geqq \eta / 2$.

From the continuity of $B$ and $S$ and from (C5)' it follows that $\delta_{i}>0$. Define $t_{i+1}=t_{i}+\delta_{i}$ and

$$
\phi(t)=S\left(t, t_{i}\right) \phi\left(t_{i}\right)+\int_{t_{i}}^{t} T(t, r) B\left(t_{i}, \phi\left(t_{i}\right)\right) d r \quad \text { for } t \in\left[t_{i}, t_{i+1}\right) .
$$

From (3) select a $\phi\left(t_{i+1}\right) \in D\left(t_{i+1}\right)$ such that 


$$
\left|\phi\left(t_{i+1}-\right)-\phi\left(t_{i+1}\right)\right| \leqq\left(t_{i+1}-t_{i}\right) \varepsilon .
$$

Clearly $\phi$ is an $\varepsilon$-approximate solution on $\left[a, t_{i+1}\right]$ and $(\varepsilon 4)$ and $(\varepsilon 5)$ hold on $\left[a, t_{i+1}\right]$ by (2). To complete the proof we show that $t_{i} \geqq \sigma$ for some $i \geqq 1$. Suppose, for contradiction, that $t_{i}<\sigma$ for all $i \geqq 0$ and let $c=\lim _{i \rightarrow \infty} t_{i}$. Let $\phi$ be defined by (2.2) and note that $\left(t_{i} \phi\left(t_{i}\right)\right) \in F$ for all $i \geqq 0$ by Lemma 2.2. From Lemma 2.1 it follows that if $k$ is a positive integer,

$$
\limsup _{i, j \rightarrow \infty}\left|\phi\left(t_{i}\right)-\phi\left(t_{j}\right)\right| \leqq 2 M\left(c-t_{k}\right) \varepsilon+\limsup _{i, j \rightarrow \infty}\left|\psi\left(t_{i}, t_{k}\right)-\psi\left(t_{j}, t_{k}\right)\right| \text {. }
$$

Since $|B(\gamma(r), \phi(\gamma(r)))| \leqq P$ for $r \in[a, c)$ it follows that

and hence

$$
\begin{aligned}
\left|\psi\left(t_{i}, t_{k}\right)-\psi\left(t_{j}, t_{k}\right)\right| \leqq & \left|S\left(t_{i}, t_{k}\right) \phi\left(t_{k}\right)-S\left(t_{j}, t_{k}\right) \phi\left(t_{k}\right)\right| \\
& +\left(t_{i}-t_{k}\right) M \cdot P+\left(t_{j}-t_{k}\right) M \cdot P,
\end{aligned}
$$

$$
\limsup _{i, j \rightarrow \infty}\left|\phi\left(t_{i}\right)-\phi\left(t_{j}\right)\right| \leqq 2 M\left(c-t_{k}\right) \varepsilon+2\left(c-t_{k}\right) M \cdot P
$$

for each $k \geqq 1$. Letting $k \rightarrow \infty$ shows that $w=\lim _{i \rightarrow \infty} \phi\left(t_{i}\right)$ exists and $(c, w) \in F$ since $F$ is closed. From the relative compactness of $\left\{\left(t_{i}, \phi\left(t_{i}\right)\right): i \geqq 0\right\}$ it is easy to see that there is a $\delta>0$ and a $k \geqq 1$ such that (1) and (2) above hold with $\delta_{i}$ replaced by $\left(c+\nu-t_{i}\right)$ for each $\nu \in(0, \delta]$ and $i \geqq k$. Thus, using (4) and (3), we have that if $\nu \in(0, \delta]$ and $i \geqq k$ is sufficiently large so that $2 \delta_{i}<\nu$,

$$
d\left(S\left(c+\nu, t_{i}\right) \phi\left(t_{i}\right)+\int_{t_{i}}^{c+\nu} T(c+\nu, r) B\left(t_{i}, \phi\left(t_{i}\right)\right) d r ; D(c+\nu)\right)>\left(c+\nu-t_{i}\right) \varepsilon / 2 .
$$

Letting $i \rightarrow \infty$ we obtain that

$$
d\left(S(c+\nu, c) w+\int_{c}^{c+\nu} T(c+\nu, r) B(c, w) d r ; D(c+\nu)\right) \geqq \nu \varepsilon / 2 .
$$

Since this is true for all $\nu \in(0, \delta]$ we have a contradiction to (C5)'. Thus $t_{i} \geqq \sigma$ for some $i \geqq 1$ and the proof of Proposition 2.1 is complete.

Under the conditions ( 1 )-(C5) we may construct $\varepsilon$-approximate solutions to (1.4) for each $0<\varepsilon \leqq \varepsilon_{0}$. For convenience of future reference we list the following suppositions and notations :

(D1) Conditions (C1)-(C5) are fulfilled;

(D2) $\left\{\varepsilon_{n}\right\}_{1}^{\infty}$ is a sequence in $\left(0, \varepsilon_{0}\right]$ such that $\lim _{n \rightarrow \infty} \varepsilon_{n}=0$;

(D3) for each $n \geqq 1, \rho_{n} \in\left[\sigma, \sigma+\varepsilon_{0}\right)$ and $\phi_{n}$ is an $\varepsilon_{n}$-approximate solution to (1.4) on $\left[0, \rho_{n}\right]$ which satisfies $(\varepsilon 1)-(\varepsilon 5)$ with $\varepsilon$ replaced by $\varepsilon_{n}$; and

(D4) for each $n \geqq 1,\left\{t_{i}^{n}\right\}_{i=0}^{N(n)}$ is the partition of $\left[0, \rho_{n}\right]$ corresponding to $\phi_{n}$, and the functions $\gamma_{n}$ and $\phi_{n}$ correspond to $\phi_{n}$ as indicated by $\gamma$ in 
(2.1) and by $\phi$ in (2.2), respectively.

Proposition 2.2. Suppose that (D1)-(D4) hold and that $u(t)=\lim _{n \rightarrow \infty} \phi_{n}(t)$ exists uniformly for $t \in[0, \sigma]$. Then $u$ is a solution to (1.4) on $[0, \sigma]$.

PROoF. Since $\left|\psi_{n}(t, a)-\phi_{n}(t)\right| \leqq M(\sigma-a) \varepsilon_{n}$ for $n \geqq 1$ by Lemma 2.1, $\phi_{n}(t, a)$ $\rightarrow u(t)$ uniformly on $[0, \sigma]$. Thus $u$ is continuous (since each $\phi_{n}(\cdot, a)$ is continuous). Moreover, if $t \in[0, \sigma]$ we have from $(\varepsilon 4)$ that

$$
\begin{aligned}
\mid u(t) & -\phi_{n}\left(\gamma_{n}(t)\right)|\leqq| u(t)-\phi_{n}(t) \mid \\
& +\left|S\left(t, \gamma_{n}(t)\right) \phi_{n}\left(\gamma_{n}(t)\right)+\int_{\gamma_{n}(t)}^{t} T(t, r) B\left(\gamma_{n}(r), \phi_{n}\left(\gamma_{n}(r)\right)\right) d r-\phi_{n}\left(\gamma_{n}(t)\right)\right| \\
& \leqq \\
\quad & \leqq \\
\quad & \left|u(t)-\phi_{n}(t)\right|+\varepsilon_{n}+\left(t-\gamma_{n}(t)\right) M \cdot P \\
&
\end{aligned}
$$

and hence $u(t)=\lim _{n \rightarrow \infty} \phi_{n}\left(\gamma_{n}(t)\right)$ uniformly for $t \in[0, \sigma]$. Since $\left(\gamma_{n}(t), \phi_{n}\left(\gamma_{n}(t)\right)\right) \in F$ and $F$ is closed it follows that $(t, u(t)) \in F$, and hence $u(t) \in D(t)$ for all $t \in[0, \sigma]$. Since $\{(t, u(t)): t \in[0, \sigma]\}$ is compact and $B$ is continuous on $D$, one also has that $B\left(\gamma_{n}(r), \phi_{n}\left(\gamma_{n}(r)\right)\right) \rightarrow B(r, u(r))$ uniformly on $[0, \sigma]$. Therefore,

$$
\begin{aligned}
& u(t)-S(t, a) z-\int_{a}^{t} T(t, r) B(r, u(r)) d r \\
& =\lim _{n \rightarrow \infty}\left\{\phi_{n}(t, a)-S(t, a) z-\int_{a}^{t} T(t, r) B\left(\gamma_{n}(r), \phi_{n}\left(\gamma_{n}(r)\right)\right) d r\right\} \\
& =\theta,
\end{aligned}
$$

and the proof is complete.

Condition (C5) is intimately related to the existence of approximate solutions to (1.4). For each $c \in[a, b)$ consider the integral equation

$$
u(t)=S(t, c) z+\int_{c}^{t} T(t, r) B(r, u(r)) d r \quad \text { for } t \in[c, \sigma] \subset[c, b)
$$

where $z \in D(c)$. The definitions of solution and $\varepsilon$-approximate to (2.3) are completely analogous to those for (1.4) with $[a, b)$ replaced by $[c, b)$. There is the following connection between the existence of approximate solutions to (2.3) and the condition (C5).

Proposition 2.3. Let $D, S, T$ and $B$ be as in $\$ 1$. Then these are equivalent:

(i) $\liminf _{h \rightarrow 0+} d(S(c+h, c) z+h B(c, z) ; D(c+h)) / h=0$ for all $(c, z) \in D$.

(ii) For each $(c, z) \in D$ and $\varepsilon>0$ there is a $\sigma=\sigma(c, z, \varepsilon) \in(c, b)$ such that (2.3) has an $\varepsilon$-approximate solution $\phi=\phi_{c, z, \varepsilon}$ on $[c, \sigma]$.

Moreover, if (i) holds the number $\sigma=\sigma(c, z, \varepsilon)$ in (ii) may be chosen independent 
of $\varepsilon>0$.

Proof. The fact that (i) implies (ii) and $\sigma(c, z, \varepsilon)$ is independent of $\varepsilon>0$ follows from Proposition 2.1. Now suppose that (ii) holds, $(c, z) \in D$ and $\varepsilon>0$. Let $\left\{t_{i}\right\}_{1}^{N}$ be the partition of $[c, \sigma]$ corresponding to $\phi=\phi_{c, z, \varepsilon}$. Then $0<t_{1}-c$ $<\varepsilon$ and $\phi\left(t_{1}\right) \in D\left(t_{1}\right)$, so from $(\varepsilon 1)-(\varepsilon 3)$,

Since

$$
\begin{gathered}
d\left(S\left(t_{1}, c\right) z+\int_{c}^{t_{1}} T\left(t_{1}, r\right) B(c, z) d r ; D\left(t_{1}\right)\right)=d\left(\phi\left(t_{1}-\right) ; D(t)\right) \\
\leqq\left|\phi\left(t_{1}-\right)-\phi\left(t_{1}\right)\right| \leqq\left(t_{1}-c\right) \varepsilon .
\end{gathered}
$$

$$
\left(t_{1}-c\right) B(c, z)=\int_{c}^{t_{1}} T\left(t_{1}, r\right) B(c, z) d r+o\left(t_{1}-c\right)
$$

where $h^{-1}|o(h)| \rightarrow 0$ as $h \rightarrow 0+$, it follows easily that (i) holds and the proof is complete.

REMARK 2.1. One can actually show that if (i) in Proposition 2.3 holds then it necessarily follows that

$$
\lim _{h \rightarrow 0+} d(S(c+h, c) z+h B(c, z) ; D(c+h)) / h=0,
$$

and this limit is uniform for $(c, z)$ in each compact subset of $D$. However, the proof of this fact uses a more complicated definition of $\varepsilon$-approximate solution (see [1]).

A convenient criteria that one may use to insure that (i) in Proposition 2.3 holds is provided by the following result.

Proposition 2.4. Suppose that $E$ is a closed subset of $X, D=[a, b) \times E$, and that

(a) $S(t, s): E \rightarrow E$ for all $a \leqq s \leqq t<b$, and

(b) $\quad \liminf _{h \rightarrow 0+} d(x+h B(t, x) ; E) / h=0$ for all $(t, x) \in D$.

Then (i) in Proposition 2.3 holds.

Proof. By [1, Theorem 2] it follows that

$$
\lim _{h \rightarrow 0+} d(x+h B(t, x) ; E) / h=0
$$

uniformly for $(t, x)$ in each compact subset of $[a, b) \times E$ (see also [2, Lemma $1])$. Let $(c, z) \in[a, b) \times E$ and choose $\delta>0$ such that $c+\delta<b$. Then

$$
K=\{S(c+h, c) z: 0 \leqq h \leqq \delta\}
$$

is a compact subset of $E$ by (a). Since

$$
S(c+h, c) z+h B(c, z)=S(c+h, c) z+h B(c, S(c+h, c) z)+o(h)
$$

where $h^{-1}|o(h)| \rightarrow 0$ as $h \rightarrow 0+$, it follows that 


$$
\begin{aligned}
& d(S(c+h, c) z+h B(c, z) ; E) / h \\
& \leqq d(S(c+h, c) z+h B(c, S(c+h, c) z) ; E) / h+h^{-1}|o(h)| \\
& \leqq \sup _{y \in K}\left\{d(y+h B(c, y) ; E) / h+h^{-1}|o(h)|\right\} \\
& \rightarrow 0 \text { as } h \rightarrow 0+.
\end{aligned}
$$

Thus (i) in Proposition 2.3 is valid.

REMARK 2.2. The conditions (a) and (b) in Proposition 2.4 are analogous to those used in [2].

\section{§ 3. Existence of solutions.}

The notations of the previous two sections are continued in this section. First we consider two conditions which guarantee the local existence of solutions to (1.4).

THEOREM 3.1. In addition to conditions (C1)-(C5) suppose that $T(t, s)$ is a compact linear operator for each $a \leqq s<t<b$. Then (1.4) has a solution $u$ on $[a, \sigma]$ with $u(t) \in D(t)$ for each $t \in[a, \sigma]$.

Proof. Let (D1)-(D4) of the previous section be valid. From Lemma 2.1 and Proposition 2.2 it suffices to show that the sequence $\left\{\psi_{n}(\cdot, a)\right\}_{1}^{\infty}$ has a uniformly convergent subsequence on $[a, \sigma]$. Using Ascoli's theorem we show that $\left\{\psi_{n}(t, a): n \geqq 1\right\}$ has compact closure for each $t \in[a, \sigma]$ and that $\left\{\psi_{n}(\cdot, a)\right\}_{1}^{\infty}$ is equicontinuous on $[a, \sigma]$. For notational convenience set

$$
V_{n}(r)=B\left(\gamma_{n}(r), \phi_{n}\left(\gamma_{n}(r)\right)\right)
$$

for $r \in\left[a, \rho_{n}\right]$ and $n \geqq 1$. Clearly $\left\{\psi_{n}(a, a)\right\}_{1}^{\infty}$ is compact, so let $a<t \leqq \sigma$ and let $0<\varepsilon<t-a$. Noting that

$$
\phi_{n}(t, a)=S(t, a) z+T(t, t-\varepsilon) \int_{a}^{t-\varepsilon} T(t-\varepsilon, r) V_{n}(r) d r+\int_{t-\varepsilon}^{t} T(t, r) V_{n}(r) d r
$$

and

$$
\left|\int_{t-\varepsilon}^{t} T(t, r) V_{n}(r) d r\right| \leqq \varepsilon M \cdot P
$$

for all $n \geqq 1$, it follows easily that $\left\{\psi_{n}(t, a): n \geqq 1\right\}$ has compact closure since $T(t, t-\varepsilon)$ is a compact operator. It is easy to check that $\left\{\psi_{n}(t, a)\right\}_{1}^{\infty}$ is equicontinuou $_{\mathrm{S}}$ for $t=a$, so suppose $a<t \leqq \sigma$ and $\varepsilon>0$. Let $\eta>0$ be such that $a+3 \eta \leqq t$ and let $s \in[a, \sigma]$ with $|t-s| \leqq \eta$. Then $a+2 \eta \leqq s$ and

$$
\begin{array}{r}
\left|\psi_{n}(t, a)-\psi_{n}(s, a)\right| \leqq|S(t, a) z-S(s, a) z|+\left|\int_{t-2 \eta}^{t} T(t, r) V_{n}(r) d r\right| \\
+\left|\int_{t-2 \eta}^{s} T(s, r) V_{n}(r) d r\right|+\left|\int_{a}^{t-2 \eta}[T(t, r)-T(s, r)] V_{n}(r) d r\right|
\end{array}
$$


for each $n \geqq 1$. Since the first (resp., second) integral on the right is bounded by $2 \eta M \cdot P$ (resp., $3 \eta M \cdot P$ ) we may assume that $\eta$ is sufficiently small so that

$$
\left|\psi_{n}(t, a)-\psi_{n}(s, a)\right| \leqq 3 \varepsilon / 4+\left|\int_{a}^{t-2 \eta}[T(t, r)-T(s, r)] V_{n}(r) d r\right|
$$

for all $n \geqq 1$ and $s \in[a, \sigma]$ with $|t-s| \leqq \eta$. The set

$$
K \equiv\left\{T(t-\eta, t-2 \eta) T(t-2 \eta, r) V_{n}(r): n \geqq 1 \text { and } a \leqq r \leqq t-2 \eta\right\}
$$

has compact closure, so the map $(\xi, x) \rightarrow T(\xi, t-\eta) x$ is uniformly continuous from $[t-\eta, \sigma] \times K$ into $X$. Noting that

$$
[T(t, r)-T(s, r)] V_{n}(r)=[T(t, t-\eta)-T(s, t-\eta)] \cdot T(t-\eta, r) V_{n}(r)
$$

where $T(t-\eta, r) V_{n}(r) \in K$ for all $r \in[a, t-2 \eta]$, one sees that there is a $\delta=$ $\delta(\varepsilon)>0$ such that $\delta \leqq \eta$ and

$$
\left|\int_{a}^{t-2 \eta}[T(t, r)-T(s, r)] V_{n}(r) d r\right| \leqq \varepsilon / 4
$$

whenever $|t-s| \leqq \delta$ and $n \geqq 1$. This establishes the equicontinuity of $\left\{\psi_{n}(t, a)\right\}_{1}^{\infty}$ and completes the proof of Theorem 3.1.

One may also place a Lipschitz condition on $B$ to obtain local existence criteria.

THEOREM 3.2. In addition to conditions (C1)-(C5) suppose that there is a number $L>0$ such that at least one of the following two conditions are satisfied. (a) $D(t)=D(a)$ for all $t \in[a, b)$ and $|B(t, x)-B(t, y)| \leqq L|x-y|$ for all $(t, x)$, $(t, y) \in F$.

(b) There is a continuous function $\nu:[0, b-a) \rightarrow[0, \infty)$ such that $\nu(0)=0$ and

$$
|B(t, x)-B(s, y)| \leqq L|x-y|+\nu(|t-s|) \text { for all }(t, x),(s, y) \in F .
$$

Then (1.4) has a unique solution $u$ on $[a, \sigma]$ with $u(t) \in D(t)$ for all $t \in[a, \sigma]$.

ProOF. Let (D1)-(D4) of the previous section be valid. To establish existence we show that $\left\{\psi_{n}(\cdot, a)\right\}_{1}^{\infty}$ converges uniformly on $[a, \sigma]$ (see Lemma 2.1 and Proposition 2.2). Using $(\varepsilon 4)$ it follows that

$$
\begin{aligned}
\left|\phi_{n}(t)-\phi_{n}\left(\gamma_{n}(t)\right)\right| \leqq & \left|S\left(t, \gamma_{n}(t)\right) \phi_{n}\left(\gamma_{n}(t)\right)-\phi_{n}\left(\gamma_{n}(t)\right)\right| \\
& +\int_{\gamma_{n}(t)}^{t}\left|T(t, r) B\left(\gamma_{n}(r), \phi_{n}\left(\gamma_{n}(r)\right)\right)\right| d r \\
\leqq & \varepsilon_{n}(1+M \cdot P),
\end{aligned}
$$

and from Lemma 2.1 there is an $N_{1}>0$ such that

$$
\left|\phi_{n}(t, a)-\phi_{n}\left(\gamma_{n}(t)\right)\right| \leqq N_{1} \varepsilon_{n} \text { for all } n \geqq 1 \text { and } t \in[a, \sigma] \text {. }
$$

Now let $n$ and $m$ be positive integers and let $r \in(a, \sigma]$. Set $t_{i}^{n}=\gamma_{n}(r)$ and $t_{j}^{m}=$ $\gamma_{m}(r)$ and assume for definiteness that $t_{i}^{n} \leqq t_{j}^{m}$. Then $\gamma_{m}(r) \in\left[t_{i}^{n}, t_{i+1}^{n}\right)$ so if as- 
sumption (a) holds we have from ( 85$)$ that

$$
\left|B\left(\gamma_{n}(r), \phi_{n}\left(\gamma_{n}(r)\right)\right)-B\left(\gamma_{m}(r), \phi_{n}\left(\gamma_{n}(r)\right)\right)\right| \leqq \varepsilon_{n} .
$$

Therefore,

$$
\begin{aligned}
& \left|B\left(\gamma_{n}(r), \phi_{n}\left(\gamma_{n}(r)\right)\right)-B\left(\gamma_{m}(r), \phi_{m}\left(\gamma_{m}(r)\right)\right)\right| \\
& \quad \leqq\left|B\left(\gamma_{m}(r), \phi_{n}\left(\gamma_{n}(r)\right)\right)-B\left(\gamma_{m}(r), \phi_{m}\left(\gamma_{m}(r)\right)\right)\right|+\varepsilon_{n} \\
& \quad \leqq L \cdot\left|\phi_{n}\left(\gamma_{n}(r)\right)-\phi_{m}\left(\gamma_{m}(r)\right)\right|+\varepsilon_{n},
\end{aligned}
$$

and it follows from (3.1) that if (a) holds there is a continuous function $\delta$ : $[0, \infty) \rightarrow[0, \infty)$ such that $\delta(0)=0$ and

$$
\begin{aligned}
& \left|B\left(\gamma_{n}(r), \phi_{n}\left(\gamma_{n}(r)\right)\right)-B\left(\gamma_{m}(r), \phi_{m}\left(\gamma_{m}(r)\right)\right)\right| \\
& \leqq L \cdot\left|\phi_{n}(r, a)-\phi_{m}(r, a)\right|+\delta\left(\varepsilon_{n}+\varepsilon_{m}\right)
\end{aligned}
$$

for all $n, m \geqq 1$ and $r \in[a, \sigma]$. Since $\left|\gamma_{n}(r)-\gamma_{m}(r)\right| \leqq \varepsilon_{n}+\varepsilon_{m}$ for all $n, m \geqq 1$ and $r \in[a, \sigma]$ one may easily check that there is also a function $\delta$ such that (3.2) is valid when (b) holds as well. If $n$ and $m$ are positive integers and $p(t)=$ $\left|\psi_{n}(t, a)-\psi_{m}(t, a)\right|$ for all $t \in[a, \sigma]$, then $p$ is continuous and from $(3.2)$ it follows that

$$
\begin{aligned}
p(t) & =\left|\int_{a}^{t} T(t, r)\left[B\left(\gamma_{n}(r), \phi_{n}\left(\gamma_{n}(r)\right)\right)-B\left(\gamma_{m}(r), \phi_{m}\left(\gamma_{m}(r)\right)\right)\right] d r\right| \\
& \leqq \int_{a}^{t} M \cdot\left[L p(r)+\delta\left(\varepsilon_{n}+\varepsilon_{m}\right)\right] d r \\
& \leqq(\sigma-a) M \delta\left(\varepsilon_{n}+\varepsilon_{m}\right)+\int_{a}^{t} M \cdot L p(r) d r
\end{aligned}
$$

for all $t \in[a, \sigma]$. From Gronwall's inequality,

$$
\left|\psi_{n}(t, a)-\psi_{m}(t, a)\right| \leqq(\sigma-a) M \delta\left(\varepsilon_{n}+\varepsilon_{m}\right) e^{M L(t-a)}
$$

for all $n, m \geqq 1$ and $t \in[a, \sigma]$, and hence $\left\{\psi_{n}(t, a)\right\}_{1}^{\infty}$ is uniformly Cauchy on $[a, \sigma]$. This establishes the existence of a solution $u$ and the uniqueness assertion follows routinely from Gronwall's inequality.

Theorems 3.1 and 3.2 provide criteria for the local existence of solutions to (1.4). Given the local existence of solutions one may also obtain somewhat usual criteria for the global existence of solutions. For each $(c, z) \in D$ consider the initial value problem

$$
u(t)=S(t, c) z+\int_{c}^{t} T(t, r) B(r, u(r)) d r, \quad t \in[c, b) .
$$

The following result may be obtained using the usual techniques and we omit its proof.

Proposition 3.1. Suppose that $D_{s} \equiv\{(t, x) \in D: a \leqq t \leqq s\}$ is closed for each 
$s \in[a, b), B$ maps bounded subsets of $D_{s}$ into bounded subsets of $X$ for each $s \in[a, b)$, and (3.3) has a local solution for each $(c, z) \in D$. If $(c, z) \in D$ and $u_{c, z}$ is a noncontinuable solution to (3.3) then $u_{c, z}$ is defined on a subinterval of $[c, b$ ) having the form $[c, \sigma)$ for some $\sigma=\sigma_{c, z} \in(c, b]$. Moreover, if $\sigma<b$ then

$$
\lim _{t \rightarrow \sigma-}\left|u_{c, z}(t)\right|=\infty \text {. }
$$

REMARK 3.1. If one has a precise exponential growth bound on the linear system $T$ (i.e., $\|T(t, s)\| \leqq e^{\omega(t-s)}$ for all $a \leqq s \leqq t<b$, and some number $\boldsymbol{\omega} \in \boldsymbol{R})$, then the Lipschitz condition on the nonlinear term $B$ in Theorem 3.2 may be relaxed. In this case one may assume a dissipative or one-side estimate on $B$ (i.e., $|x-y-h[B(t, x)-B(t, y)]| \geqq(1-h L)|x-y|$ for all $h>0$ and $(t, x)$, $(t, y) \in D(B))$. However, the arguments become quite long and tedious and we will not consider this situation.

REMARK 3.2. Each of the results in this and the preceding section is valid if one only assumes that $\alpha$ is continuous from $[a, b)$ into $X$ as opposed to continuously differentiable from $[a, b)$ into $X$. There are no changes in the proofs. However, the differentiability of $\alpha$ was used to connect $S$ with the generator of $T$ in section 1 (see (1.5)).

\section{§4. Inequalities.}

In this section we indicate how these techniques may be employed to obtain relationships involving inequalities for solutions to abstract differential equations. Throughout this section it is supposed that $C$ is a closed cone in $X$ (i.e., $C$ is a closed subset of $X$ and $x+y, \alpha x \in C$ whenever $x, y \in C$ and $\alpha \geqq 0$ ) and write $x \geqq y$ (or $y \leqq x$ ) whenever $x, y \in X$ with $x-y \in C$. Also, it is supposed that for $i=1,2$, the families $T_{i}=\left\{T_{i}(t, s):(t, s) \in \Delta\right\}$ and $S_{i}=\left\{S_{i}(t, s):(t, s) \in \Delta\right\}$ possess each of the properties of $T$ and $S$ in $\S 1$. Moreover, for $i=1,2$, it is supposed that $D_{i}$ is a locally closed subset of $[a, b) \times X$ having each of the properties of $D$ in $\S 1$ and that $B_{i}$ is a continuous function from $D\left(B_{i}\right) \subset \boldsymbol{R} \times X$ into $X$ such that $D\left(B_{i}\right) \supset D_{i}$. Here we consider the pair of initial value problems

$$
\begin{aligned}
& u_{1}(t)=S_{1}(t, c) z_{1}+\int_{c}^{t} T_{1}(t, r) B_{1}\left(r, u_{1}(r)\right) d r \\
& u_{2}(t)=S_{2}(t, c) z_{2}+\int_{c}^{t} T_{2}(t, r) B_{2}\left(r, u_{2}(r)\right) d r
\end{aligned} \quad t \in[c, b)
$$

where $z_{1} \in D_{1}(c)$ and $z_{2} \in D_{2}(c)$. Instead of assuming that $T_{i}$ is compact or that $B_{i}$ is locally Lipschitz, we make the following basic assumption on the existence of solutions to (4.1): 


$$
\left\{\begin{array}{l}
\text { If } i \in\{1,2\},\left(c, z_{i}\right) \in D_{i} \text { and } \phi_{n}^{i} \text { is an } \varepsilon_{n} \text {-approximate } \\
\text { solution to the } i^{t h} \text { equation in (4.1) on }\left[c, \rho_{n}\right] \text { for each } \\
n \geqq 1 \text {, where } \varepsilon_{n} \rightarrow 0 \text { as } n \rightarrow \infty \text { and inf }\left\{\rho_{n}-c: n \geqq 1\right\}>0, \\
\text { then }\left\{\phi_{i}^{n}\right\}_{1}^{\infty} \text { has a uniformly convergent subsequence } \\
\text { on }[c, \sigma] \text { for some } \sigma \in(c, b) .
\end{array}\right.
$$

Under these suppositions and notations we consider the following problem: If $\left(c, z_{1}\right) \in D_{1}$ and $\left(c, z_{2}\right) \in D_{2}$ with $z_{1} \geqq z_{2}$, is there a $\sigma \in(c, b)$ and a solution $\left(u_{1}, u_{2}\right)$ to (4.1) on $[c, \sigma]$ such that $u_{1}(t) \geqq u_{2}(t)$ for all $t \in[c, \sigma]$ ?

One may obtain criteria insuring an affirmative answer to this question directly from the results of $\S 2$. Let $\mathscr{X}$ be the Banach space $X \times X$ with $\left|\left(x_{1}, x_{2}\right)\right|=\left|x_{1}\right|+\left|x_{2}\right|$ for all $\left(x_{1}, x_{2}\right) \in \mathscr{X}$, and define the families $\mathscr{I}$ and $\mathcal{S}$ of mappings from $\mathscr{X}$ into $\mathscr{X}$ by

and

$$
\mathscr{T}(t, s)\left(x_{1}, x_{2}\right)=\left(T_{1}(t, s) x_{1}, T_{2}(t, s) x_{2}\right)
$$

$$
\mathcal{S}(t, s)\left(x_{1}, x_{2}\right)=\left(S_{1}(t, s) x_{1}, S_{2}(t, s) x_{2}\right)
$$

for all $(t, s) \in \Delta$ and $\left(x_{1}, x_{2}\right) \in \mathscr{X}$. Also, define

$$
D(\mathscr{B})=\left\{\left(t, x_{1}, x_{2}\right):\left(t, x_{1}\right) \in D\left(B_{1}\right),\left(t, x_{2}\right) \in D\left(B_{2}\right)\right\}
$$

and

$$
\mathcal{B}\left(t, x_{1}, x_{2}\right)=\left(B_{1}\left(t, x_{1}\right), B_{2}\left(t, x_{2}\right)\right) \quad \text { for all }\left(t, x_{1}, x_{2}\right) \in D(B) .
$$

Note that (4.1) is equivalent to the initial value problem

$$
\Phi(t)=\mathcal{S}(t, c)\left(z_{1}, z_{2}\right)+\int_{c}^{t} \mathscr{I}(t, r) \mathscr{B}(r, \Phi(r)) d r, \quad t \in[c, b)
$$

in $\mathscr{X}$, where $\Phi(t)=\left(u_{1}(t), u_{2}(t)\right)$. Defining

$$
Q=\left\{\left(t, x_{1}, x_{2}\right):\left(t, x_{1}\right) \in D_{1},\left(t, x_{2}\right) \in D_{2} \quad \text { and } \quad x_{1} \geqq x_{2}\right\}
$$

one sees that $Q$ is a locally closed subset of $\mathscr{X}$, and our question has an affirmative answer only in case there is a solution $\Phi$ to (4.3) on $[c, \sigma]$ such that $\Phi(t) \in Q(t)$ for all $t \in[c, \sigma]$, where $Q(t)=\left\{\left(x_{1}, x_{2}\right) \in \mathscr{X}:\left(t, x_{1}, x_{2}\right) \in Q\right\}$. Letting $d(\psi ; \mathcal{E})=\inf \{|\psi-x|: x \in \mathcal{E}\}$ for each $\phi \in \mathscr{X}$ and $\mathcal{E} \subset \mathfrak{X}$, we obtain the following result directly from Proposition 2.1 and 2.2 and assumption (4.2):

Proposition 4.1. Let each of the above suppositions and notations hold and suppose that

$$
\liminf _{h \rightarrow 0+} d(\mathcal{S}(c+h, c) \phi+h \mathscr{B}(c, \phi) ; Q(c+h)) / h=0
$$

for all $(c, \phi) \in Q$. Then for each $\left(c, z_{1}\right) \in D_{1}$ and $\left(c, z_{2}\right) \in D_{2}$ with $z_{1} \geqq z_{2}$ there is a $\sigma=\sigma\left(c, z_{1}, z_{2}\right) \in(c, b)$ and a solution $\left(u_{1}, u_{2}\right)$ to (4.1) on $[c, \sigma]$ such that $\left(u_{1}(t)\right.$, $\left.u_{2}(t)\right) \in Q(t)$, and hence $u_{1}(t) \geqq u_{2}(t)$ for all $t \in[c, \sigma]$. 
Now suppose that $D_{1}$ and $D_{2}$ have the property that for each $\left(t, x_{1}\right) \in D_{1}$ there is a $\left(t, x_{2}\right) \in D_{2}$ such that $x_{1} \geqq x_{2}$ and for each $\left(t, x_{2}\right) \in D_{2}$ there is a $\left(t, x_{1}\right) \in D_{1}$ such that $x_{1} \geqq x_{2}$. One may easily check that (4.4) implies the following :

$$
\left\{\begin{array}{l}
\text { For each }\left(c, x_{1}\right) \in D_{1} \text { and }\left(c, x_{2}\right) \in D_{2} \text { with } x_{1} \geqq x_{2} \text { there } \\
\text { is a sequence }\left\{h_{n}\right\}_{1}^{\infty} \text { with } h_{n} \rightarrow 0 \text { as } n \rightarrow \infty \text { such that } \\
\lim _{n \rightarrow \infty} d\left(S_{i}\left(c+h_{n}, c\right) x_{i}+h_{n} B_{i}\left(c, x_{i}\right) ; D_{i}\left(c+h_{n}\right)\right) / h_{n}=0 \text { for } \\
i=1,2 \text {, and } \lim _{n \rightarrow \infty} d\left(S_{1}\left(c+h_{n}, c\right) x_{1}-S_{2}\left(c+h_{n}, c\right) x_{2}+h_{n}\left[B_{1}(c,\right.\right. \\
\left.\left.\left.x_{1}\right)-B_{2}\left(c, x_{2}\right)\right] ; C\right) / h_{n}=0 .
\end{array}\right.
$$

It does not seem to be known if (4.5) and (4.4) are equivalent. Under certain additional assumptions the equivalence of (4.4) and (4.5) may be established. One such assumption involving $D_{1}, D_{2}$ and $C$ is the following:

(Q) There is a number $N>0$ such that if $K_{i}$ is a compact subset of $D_{i}$ for $i=1,2$ there is a number $\eta=\eta\left(K_{1}, K_{2}\right)$ with the following property: if $\left(t, x_{i}\right) \in K_{i}$ with $d\left(x_{1}-x_{2} ; C\right) \leqq \eta$, there is a $\left(t, \bar{x}_{2}\right) \in D_{2}$ such that $x_{1} \geqq \bar{x}_{2}$ and $\left|x_{2}-\bar{x}_{2}\right| \leqq N d\left(x_{1}-x_{2} ; C\right)$.

When this condition is satisfied we say that $\left(D_{1}, D_{2}, C\right)$ satisfy $(\mathrm{Q})$. It is easy to check that if $D_{1}$ and $D_{2}$ are open then $\left(D_{1}, D_{2}, C\right)$ satisfy $(\mathrm{Q})$.

Proposition 4.2. Suppose that $\left(D_{1}, D_{2}, C\right)$ satisfy (Q) and that (4.5) is valid. Then (4.4) holds.

PROOF. Let $\left(c, x_{1}, x_{2}\right) \in Q$ and set $x_{i}(h)=S_{i}(c+h, c) x_{i}+h B_{i}\left(c, x_{i}\right)$ for $h>0$ and $i=1,2$. From (4.5) we have that $d\left(x_{1}\left(h_{n}\right)-x_{2}\left(h_{n}\right) ; C\right)=o\left(h_{n}\right)$ and that there is a $y_{n}^{i} \in D_{i}\left(c+h_{n}\right)$ such that

$$
\left|x_{i}\left(h_{n}\right)-y_{n}^{i}\right|=o\left(h_{n}\right) \text { for } n \geqq 1 \text { and } i=1,2 \text {. }
$$

Since $y_{n}^{i} \rightarrow x_{i}$ as $n \rightarrow \infty$, there is a compact subset $K_{i}$ of $D_{i}$ such that $\left(c+h_{n}, y_{n}^{i}\right)$ $\in K_{i}$ for $n \geqq 1$ and $i=1,2$. By (Q) there is a $\bar{y}_{n}^{2} \in D_{2}\left(c+h_{n}\right)$ such that $y_{n}^{1} \geqq \bar{y}_{n}^{2}$ and $\left|y_{n}^{2}-\bar{y}_{n}^{2}\right|=o\left(h_{n}\right)$. Hence $\left(y_{n}^{1}, \bar{y}_{n}^{2}\right) \in Q\left(c+h_{n}\right)$ and

$$
\begin{aligned}
d\left(\mathcal { S } ( c + h _ { n } , c ) \left(x_{1},\right.\right. & \left.\left.x_{2}\right)+h_{n} \mathcal{B}\left(c, x_{1}, x_{2}\right) ; Q\left(c+h_{n}\right)\right) \\
& \leqq\left|\left(x_{1}\left(h_{n}\right), x_{2}\left(h_{n}\right)\right)-\left(y_{n}^{1}, \bar{y}_{n}^{2}\right)\right| \\
& \leqq\left|x_{1}\left(h_{n}\right)-y_{n}^{1}\right|+\left|x_{2}\left(h_{n}\right)-y_{n}^{2}\right|+\left|y_{n}^{2}-\bar{y}_{n}^{2}\right| \\
& =o\left(h_{n}\right),
\end{aligned}
$$

and it follows that (4.4) holds. 
Our next result contains a convenient criteria insuring that the final condition in (4.5) is fulfilled.

LEMMA 4.1. Suppose that for each $\left(c, x_{1}\right) \in D\left(B_{1}\right)$ and $\left(c, x_{2}\right) \in D\left(B_{2}\right)$ with $x_{1} \geqq x_{2}$, it follows that

$$
\lim _{h \rightarrow 0+} d\left(x_{1}-x_{2}+h\left[B_{1}\left(c, x_{1}\right)-B_{2}\left(c, x_{2}\right)\right] ; C\right) / h=0
$$

and that $S_{1}(t, c) x_{1} \geqq S_{2}(t, c) x_{2}$ for all $t \in[c, b)$. Suppose also that for $i \in\{1,2\}$ and $\left(c, x_{i}\right) \in D_{i}$ there is a $\delta>0$ such that $S_{i}(t, c) x_{i} \in D\left(B_{i}\right)$ for each $t \in[c, c+\delta]$. Then

$$
\lim _{h \rightarrow 0+} d\left(S_{1}(c+h, c) x_{1}-S_{2}(c+h, c) x_{2}+h\left[B_{1}\left(c, x_{1}\right)-B_{2}\left(c, x_{2}\right)\right] ; C\right] / h=0
$$

for all $\left(c, x_{1}\right) \in D_{1}$ and $\left(c, x_{2}\right) \in D_{2}$ with $x_{1} \geqq x_{2}$.

Indication of Proof. Since the cone $C$ is convex, $d(x+h y ; C) / h$ is nonincreasing as $h \rightarrow 0+$ for each $x \in C$ and $y \in X$. From this observation it follows that if $K_{i} \subset D_{i}\left(B_{i}\right)$ is compact for $i=1,2$, the limit in (4.6) is uniform for $\left(c, x_{1}\right) \in K_{1}$ and $\left(c, x_{2}\right) \in K_{2}$ with $x_{1} \geqq x_{2}$. Setting $K_{i}=\left\{\left(c, S_{i}(c+h, c) x_{i}\right): 0 \leqq h \leqq \delta\right\}$ for $i=1,2$, and noting that

$$
\begin{aligned}
& S_{1}(c+h, c) x_{1}-S_{2}(c+h, c) x_{2}+h\left[B_{1}\left(c, x_{1}\right)-B_{2}\left(c, x_{2}\right)\right] \\
= & S_{1}(c+h, c) x_{1}-S_{2}(c+h, c) x_{2}+h\left[B_{1}\left(c, S_{1}(c+h, c) x_{1}\right)-B_{2}\left(c, S_{2}(c+h, c) x_{2}\right]\right. \\
& +o(h)
\end{aligned}
$$

where $h^{-1}|o(h)| \rightarrow 0$ as $h \rightarrow 0+$, establishes the lemma.

The techniques in this section may also be applied to obtain results on differential inequalities.

Proposition 4.3. Suppose that $p:[a, b) \rightarrow X$ is continuous with $(t, p(t)) \in D\left(B_{1}\right)$ for all $t \in[a, b)$ and that each of the following are satisfied:

(a) $\lim _{h \rightarrow 0+} d\left(p(c+h)-S_{1}(c+h, c) p(c)-h B_{1}(c, p(c)) ; C\right) / h=0$ for each $c \in[a, b)$;

(b) $\quad \liminf _{h \rightarrow 0+} d\left(S_{2}(c+h, c) z+h B_{2}(c, z) ; D_{2}(c+h)\right) / h=0$ for each $(c, z) \in D_{2}$ with $z \leqq$ $p(c)$;

(c) $\lim _{h \rightarrow 0+} d\left(S_{1}(c+h, c) p(c)-S_{2}(c+h, c) x_{2}+h\left[B_{1}(c, p(c))-B_{2}\left(c, x_{2}\right)\right] ; C\right) / h=0$ for each $\left(c, x_{2}\right) \in D_{2}$ with $x_{2} \leqq p(c)$; and

(d) If $D_{0}=\{(t, p(t)): t \in[a, b)\}$ then $\left(D_{0}, D_{2}, C\right)$ satisfy (Q).

Then for each $z_{2} \in D_{2}(c)$ with $z_{2} \leqq p(c)$ there is $a \sigma \in(c, b)$ and a solution $u_{2}$ to the second equation in (4.1) such that $u_{2}(t) \in D_{2}(t)$ and $u_{2}(t) \leqq p(t)$ for all $t \in[c, \sigma]$.

Proof. Let $(-\infty, p(t)]=\{x \in X: x \leqq p(t)\}$ for each $t \in[a, b)$. From (4.2) and Propositions 2.1 and 2.2 it suffices to show that 


$$
\liminf _{h \rightarrow 0+} d\left(S_{2}(c+h, c) z+h B_{2}(c, z) ;(-\infty, p(c+h)] \cap D_{2}(c+h)\right) / h=0
$$

for each $(c, z) \in D_{2}$ with $z \leqq p(c)$. So let $(c, z) \in D_{2}$ with $z \leqq p(c)$ and by (b) let $h_{n} \rightarrow 0+$ and let $\left\{w_{n}\right\}_{1}^{\infty}$ be a sequence in $D_{2}\left(c+h_{n}\right)$ such that

$$
\left|S_{2}\left(c+h_{n}, c\right) z+h_{n} B_{2}(c, z)-w_{n}\right|=o\left(h_{n}\right) .
$$

From (a) it follows that

$$
\begin{aligned}
p\left(c+h_{n}\right)-w_{n} \geqq & S_{1}\left(c+h_{n}, c\right) p(c)-S_{2}\left(c+h_{n}, c\right) z \\
& +h_{n}\left[B_{1}(c, p(c))-B_{2}(c, z)\right]+\xi_{n}
\end{aligned}
$$

where $\left|\xi_{n}\right|=o\left(h_{n}\right)$ as $n \rightarrow \infty$. Using (c) it now follows that

$$
d\left(p\left(c+h_{n}\right)-w_{n} ; C\right)=o\left(h_{n}\right) ;
$$

so by (d) there is a $\bar{w}_{n} \in D_{2}\left(c+h_{n}\right)$ such that $p\left(c+h_{n}\right) \geqq \bar{w}_{n}$ and $\left|\bar{w}_{n}-w_{n}\right|=o\left(h_{n}\right)$. Consequently, $\bar{w}_{n} \in\left(-\infty, p\left(c+h_{n}\right)\right] \cap D_{2}\left(c+h_{n}\right)$ and it follows that

$$
\begin{aligned}
& d\left(S_{2}\left(c+h_{n}, c\right) z+h_{n} B_{2}(c, z) ;\left(-\infty, p\left(c+h_{n}\right)\right] \cap D_{2}\left(c+h_{n}\right)\right) \\
& \quad \leqq\left|S_{2}\left(c+h_{n}, c\right) z+h_{n} B_{2}(c, z)-\bar{w}_{n}\right|=o\left(h_{n}\right) .
\end{aligned}
$$

Thus (4.7) holds and the proof is complete.

Since $p(c) \in D_{1}(c)$ for all $c \in[a, b)$, Lemma 4.1 provides criteria which assures that (c) in Proposition 4.3 is valid, and condition (d) is certainly fulfilled if $\left(D_{1}, D_{2}, C\right)$ satisfy $(\mathrm{Q})$. In the case that $T_{1}$ is generated by the family $L_{1}=$ $\left\{L_{1}(t): t \in[a, b)\right\}$ then we may obtain a criteria involving a differential inequality which assures that (a) in Proposition 4.3 is valid. Let $\alpha_{1}$ and $\beta_{1}$ correspond to $S_{1}$ as $\alpha$ and $\beta$ correspond to $S$ in (1.1) and define $A_{1}=\left\{A_{1}(t): t \in\right.$ $[a, b)\}$ by

$$
A_{1}(t) x=L_{1}(t)\left(x-\alpha_{1}(t)\right)+\beta_{1}(t)+\alpha_{1}^{\prime}(t) \text { for all } t \in[a, b) \text { and } x \in D\left(A_{1}(t)\right),
$$

where $D\left(A_{1}(t)\right)=\left\{x \in X: x-\alpha_{1}(t) \in D\left(L_{1}(t)\right)\right\}$ (see (1.5)).

LEMMA 4.2. With the notations enumerated in the above paragraph, suppose that $p:[a, b) \rightarrow X$ is continuous and that $p(t) \in D_{1}(t) \cap D\left(A_{1}(t)\right)$ for all $t \in[a, b)$. Suppose further that for each $c \in[a, b)$ the right derivative $p_{+}^{\prime}(c)$ exists and that

$$
p_{+}^{\prime}(c) \geqq A_{1}(c) p(c)+B_{1}(c, p(c)) .
$$

Then (a) in Proposition 4.3 is valid.

Proof. From (1.6) we have that

$$
\begin{aligned}
{[p(c+} & \left.h)-S_{1}(c+h, c) p(c)-h B_{1}(c, p(c))\right] / h \\
& =[p(c+h)-p(c)] / h-A_{1}(c) p(c)-B_{1}(c, p(c))+h^{-1} o(h) \\
& \geqq h^{-1} \xi_{h}
\end{aligned}
$$

where $h^{-1}\left|\xi_{h}\right| \rightarrow 0$ as $h \rightarrow 0+$. The lemma now follows easily noting that 


$$
d(x ; C) / h=d\left(h^{-1} x ; C\right)
$$

for all $h>0$ and $x \in X$.

\section{$\S 5 . \quad$ An example.}

In this section an example is given to illustrate our results. The techniques here are improvements of those given in [3]. Let $N$ and $M$ be positive integers and let $\Omega$ be a bounded region in $\boldsymbol{R}^{N}$ with $\partial \Omega$ smooth. Also let $|\cdot|$ be a norm on $\boldsymbol{R}^{M}$, let $1<p<\infty$, and let $X$ be the space $\mathcal{L}^{p}\left(\Omega ; \boldsymbol{R}^{M}\right)$. Let $H^{2, p}(\Omega)$ be the space of all members of $\mathcal{L}^{p}(\Omega, \boldsymbol{R})$ having two derivatives in $\mathcal{L}^{p}(\Omega ; \boldsymbol{R})$ and define the Laplacian operator $\Delta$ on $X$ by

$$
D(\Delta)=\left\{\phi=\left(\phi_{i}\right)_{1}^{M} \in X: \phi_{i} \in H^{2, p}(\Omega) \quad \text { for } i=1, \cdots, M\right\}
$$

and

$$
\Delta \phi=\left(\Delta \phi_{i}\right)_{1}^{M} \quad \text { for all } \phi=\left(\phi_{i}\right)_{1}^{M} \in D(\Delta),
$$

where $\Delta$ also denotes the Laplacian on $\mathcal{L}^{p}(\Omega ; \boldsymbol{R})$. Further, let $H_{0}^{1}{ }^{p}(\Omega)$ be the space of all members of $\mathcal{L}^{p}(\Omega ; \boldsymbol{R})$ vanishing on $\partial \Omega$ and having one derivative in $\mathcal{L}^{p}(\Omega ; \boldsymbol{R})$, and define the linear operator $L$ on $X$ by

$$
D(L)=\left\{\phi \in D(\Delta): \phi_{i} \in H_{0}^{1}{ }^{p}(\Omega) \quad \text { for } i=1, \cdots, M\right\}
$$

and

$$
L \phi=\Delta \phi \quad \text { for all } \phi \in D(L) .
$$

It is well-known that $L$ is the generator of a compact, analytic semigroup $T^{\prime}=\left\{T^{\prime}(t): t \geqq 0\right\}$ on $X$. Setting $T(t, s)=T^{\prime}(t-s)$ for $0 \leqq s \leqq t$ we have that $T=$ $\{T(t, s): 0 \leqq s \leqq t\}$ satisfies (T1) and (T2) in $\S 1$ with $a=0$ and $b=\infty$.

Let $\alpha:[0, \infty) \times \bar{\Omega} \rightarrow \boldsymbol{R}^{M}$ be continuously differentiable with $\alpha(t, \cdot) \in D(\Delta)$ and $t \rightarrow \Delta \alpha(t, \cdot)$ continuous from $[0, \infty)$ to $X$. Also suppose $\phi \in X$ and that $f=$ $\left(f_{i}\right)_{1}^{M}:[0, \infty) \times \bar{\Omega} \times \boldsymbol{R}^{M} \rightarrow \boldsymbol{R}^{M}$ is continuous. Consider the nonlinear parabolic system

$$
\left\{\begin{array}{l}
u_{t}(t, \tau)=\Delta_{\tau} u(t, \tau)+f(t, \tau, u(t, \tau)) \\
u(t, \sigma)=\alpha(t, \sigma), u(0, \tau)=\phi(\tau)
\end{array} \quad \text { for } t>0, \tau \in \Omega, \sigma \in \partial \Omega\right.
$$

where $u:[0, \infty) \times \bar{\Omega} \rightarrow \boldsymbol{R}^{M}$ and $\Delta_{\tau} u(t, \tau) \equiv[\Delta u(t, \cdot)](\tau)$. We will consider the existence of mild solutions to (5.1).

Define the mapping $\beta$ from $[0, \infty)$ into $X$ by $\beta(t)=\Delta \alpha(t, \cdot)-\alpha_{t}(t, \cdot)$ and define the family $S_{\alpha}=\left\{S_{\alpha}(t, s): 0 \leqq s \leqq t\right\}$ by

$$
S_{\alpha}(t, s) \psi=T(t, s)[\psi-\alpha(s, \cdot)]+\int_{s}^{t} T(t, r) \beta(r) d r+\alpha(t, \cdot)
$$

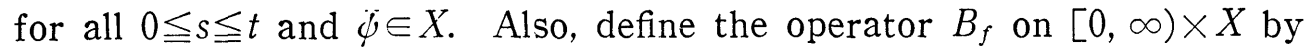




$$
\left\{\begin{array}{l}
D\left(B_{f}(t, \cdot)\right)=\{\psi \in X: \tau \rightarrow f(t, \tau, \phi(\tau)) \text { is in } X\} \text { and } \\
{\left[B_{f}(t, \phi)\right](\tau)=f(t, \tau, \phi(\tau)) \quad \text { for all } \tau \in \Omega \text { and } \phi \in D(B(t, \cdot)) .}
\end{array}\right.
$$

A function $u:[0, \infty) \times \Omega \rightarrow \boldsymbol{R}^{M}$ is said to be a mild solution to (5.1) if $u(t,) \in$ $D(B(t, \cdot))$ for all $t \geqq 0, t \rightarrow u(t, \cdot)$ is continuous from $[0, \infty)$ into $X$, and

$$
u(t, \quad)=S_{\alpha}(t, 0) \phi+\int_{0}^{t} T(t, r) B_{f}(r, u(r, \cdot)) d r \quad \text { for all } t \geqq 0
$$

(see (1.7)). We have the following basic result:

PROPOSITION 5.1. In addition to the suppositions and notations indicated above, suppose that $\Lambda$ is a closed convex subset of $\boldsymbol{R}^{M}$ such that

(a) $\lim _{h \rightarrow 0+} d(\xi+h f(t, \tau, \xi) ; \Lambda) / h=0$ for all $(t, \tau, \xi) \in[0, \infty) \times \bar{\Omega} \times \Lambda$;

(b) $a, b:[0, \infty) \rightarrow[0, \infty)$ are continuous and $|f(t, \tau, \xi)| \leqq a(t)|\xi|+b(t)$ for all $(t, \tau, \xi) \in[0, \infty) \times \bar{\Omega} \times \Lambda$; and

(c) $\alpha(t, \sigma) \in \Lambda$ for $t \geqq 0, \sigma \in \partial \Omega$, and $\phi(\tau) \in \Lambda$ for $\tau \in \Omega$.

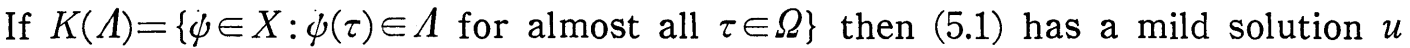
on $[0, \infty) \times \Omega$ such that $u(t, \cdot) \in K(\Lambda)$ for all $t \geqq 0$.

REMARK 5.1. Supposition (a) is equivalent to the following condition: for $(t, \tau, \xi) \in[0, \infty) \times \bar{\Omega} \times \Lambda$ the initial value problem $w^{\prime}(s)=f(t, \tau, w(s)), w(0)=\xi$ has some solution $w$ on an interval $[0, \rho)$ such that $w(s) \in \Lambda$ for all $s \in[0, \rho)$. Note also that (b) is automatically fulfilled if $\Lambda$ is bounded.

ProOF OF Proposition 5.1. From (b) one sees that $B_{f}$ is continuous from $[0, \infty) \times K(\Lambda)$ into $X$, and from (a) and [2, Proposition 5] it follows that

$$
\lim _{h \rightarrow 0+} d\left(\dot{\phi}+h B_{f}(t, \phi) ; K(\Lambda)\right) / h=0 \quad \text { for all }(t, \dot{\psi}) \in[0, \infty) \times K(\Lambda) .
$$

Since $T(t, s)$ is compact for $0 \leqq s<t$, the assertions will follow from Proposition 2.4 and Theorem 3.1 (see also Proposition 3.1) once it is shown that $S_{\alpha}(t, s)$ : $K(\Lambda) \rightarrow K(\Lambda)$. This fact follows from the maximum principle. Let $\left\{\xi_{r}^{*}: \gamma \in\right.$ $\Gamma\} \subset\left(\boldsymbol{R}^{M}\right) *$ (where $\left(\boldsymbol{R}^{M}\right)^{*}$ is the dual space of $\boldsymbol{R}^{M}$ ) and $\left\{\delta_{\gamma}: \gamma \in \Gamma\right\} \subset \boldsymbol{R}$ be such that

$$
\Lambda=\bigcap_{r \in \Gamma}\left\{\xi \in R^{M}: \xi_{r}^{*}(\xi) \leqq \delta_{r}\right\} .
$$

Let $\psi$ be a continuous member of $K(\Lambda)$, let $s \geqq 0$, and define $v(t, \tau)=\left[S_{\alpha}(t, s) \psi\right](\tau)$ for all $(t, \tau) \in[s, \infty) \times \bar{\Omega}$. From the diffierentiability of $T$ and the smoothness assumptions on $\alpha$ it follows that $v_{t}(t, \tau)$ and $\Delta_{\tau} v(t, \tau)$ exist in the classical sense for all $(t, \tau) \in(s, \infty) \times \Omega$. Thus if $\gamma \in \Gamma$ and $w(t, \tau) \equiv \xi_{r}^{*}(v(t, \tau))$ then $w_{t}(\tau, \tau)=\Delta_{\tau} w(t, \tau)$, and since $w(t, \sigma)=\xi_{r}^{*}(\alpha(t, \sigma)) \leqq \delta_{\gamma}$ for all $(t, \sigma) \in(s, \infty) \times \partial \Omega$ and $w(0, \tau)=\xi_{r}^{*}(\psi(\tau)) \leqq \delta_{r}$ for all $\tau \in \Omega$, it follows from the maximum principle that 


$$
\left.\xi_{r}^{*}(v(t, \tau))=w(t, \tau)\right) \leqq \delta_{r} \quad \text { for all }(t, \tau) \in(s, \infty) \times \Omega .
$$

Since this holds for each $\gamma \in \Gamma$ we have that $v(t, \tau) \in \Lambda$, and hence $S_{\alpha}(t, s) \dot{\phi} \in$ $K(\Lambda)$. Since $S_{\alpha}(t, s)$ is continuous and the class of continuous members $\psi$ of $K(\Lambda)$ is dense in $K(\Lambda)$, it follows that $S_{\alpha}(t, s): K(\Lambda) \rightarrow K(\Lambda)$ and the proof is complete.

The techniques of $\S 4$ may also be applied to this example. Let $R_{+}^{M}=\{\xi \in$ $\boldsymbol{R}^{M}: \xi_{i} \geqq 0$ for $\left.i=1, \cdots M\right\}$ and write $\xi \geqq \eta$ only in case $\xi-\eta \in \boldsymbol{R}_{+}^{M}$. Then

$$
K\left(\boldsymbol{R}_{+}^{M}\right)=\{\dot{\phi} \in X: \phi(\tau) \geqq \theta \text { for almost all } \tau \in \Omega\}
$$

is a closed cone in $X$, so write $\dot{\phi}_{1} \geqq \dot{\psi}_{2}$ only in case $\dot{\phi}_{1}-\phi_{2} \in K\left(\boldsymbol{R}_{+}^{M}\right)$. Now let $g=\left(g_{i}\right)_{1}^{M}:[0, \infty) \times \bar{\Omega} \times \boldsymbol{R}^{M} \rightarrow \boldsymbol{R}^{u}$ be continuous and suppose that

$$
\begin{aligned}
& \text { if } \xi \geqq \eta \geqq \theta \text { and } \xi_{i}=\eta_{i} \text { for some } i \in\{1, \cdots, M\} \text {, then } \\
& g_{i}(t, \tau, \xi) \geqq f_{i}(t, \tau, \eta) \text { for all }(t, \tau) \in[0, \infty) \times \bar{\Omega} .
\end{aligned}
$$

One may easily check that (5.5) is equivalent to

$$
\begin{aligned}
& \lim _{h \rightarrow 0+} d\left(\xi-\eta+h[g(t, \tau, \xi)-f(t, \tau, \eta)] ; \boldsymbol{R}_{+}^{M}\right) / h=0 \text { for all } \\
& (t, \tau, \xi),(t, \tau, \eta) \in[0, \infty) \times \bar{\Omega} \times \boldsymbol{R}_{+}^{M} \text { with } \xi \geqq \eta .
\end{aligned}
$$

The following result on inequalities for mild solutions to (5.1) is valid.

Proposition 5.2. Suppose that $g$ and $f$ satisfy (5.5), that $p:[0, \infty) \times \bar{\Omega} \rightarrow \boldsymbol{R}_{+}^{M}$ is continuously differentiable with $p(t, \cdot) \in D(\Delta)$ and $t \rightarrow \Delta p(t, \cdot)$ continuous from $[0, \infty)$ to $X$, and that

$$
\begin{aligned}
& p_{t}(t, \tau) \geqq \Delta_{\tau} p(t, \tau)+g(t, \tau, p(t, \tau)) \text { for all }(t, \tau) \in[0, \infty) \times \Omega \\
& \theta \leqq \phi(\tau) \leqq p(0, \tau), \theta \leqq \alpha(t, \sigma) \leqq p(t, \sigma) \text { for all } t \geqq 0, \tau \in \Omega, \sigma \in \partial \Omega .
\end{aligned}
$$

Suppose also that

$$
\begin{aligned}
& \text { if } \xi \geqq \theta \text { and } \xi_{i}=0 \text { for some } i \in\{1, \cdots, M\} \text {, then } \\
& f_{i}(t, \tau, \xi) \geqq 0 \text { for all }(t, \tau) \in[0, \infty) \times \bar{\Omega} .
\end{aligned}
$$

Then equation (5.1)] has a mild solution $u$ on $[0, \infty) \times \Omega$ such that $\theta \leqq u(t, \tau) \leqq$ $p(t, \tau)$ for all $(t, \tau) \in[0, \infty) \times \Omega$.

INDICATION OF PROOF. We show that this proposition is a consequence of Proposition 4.3. Let $S_{2}=S_{\alpha}, B_{2}=B_{f}$ and let $S_{1}$ be defined by (5.2) with $\alpha$ replaced by $p$ and let $B_{1}$ be defined by (5.3) with $f$ replaced by $g$. Since $\alpha(t, \sigma) \leqq p(t, \sigma)$ for $t \geqq 0$ and $\sigma \in \partial \Omega$, it follows that $S_{1}(t, s) \phi_{1} \geqq S_{2}(t, s) \dot{\psi}_{2}$ for all $0 \leqq s \leqq t$ and $\psi_{1}, \psi_{2} \in X$ with $\dot{\psi}_{1} \geqq \dot{\psi}_{2}$. Using (5.5) one may show that

$$
\lim _{h \rightarrow 0+} d\left(\dot{\phi}_{1}-\phi_{2}+h\left[B_{1}\left(t, \phi_{1}\right)-B_{2}\left(t, \phi_{2}\right)\right] ; K\left(\boldsymbol{R}_{+}^{M}\right)\right) / h=0
$$

for all $\dot{\phi}_{1} \in D\left(B_{1}(t, \cdot)\right), \quad \dot{\psi}_{2} \in D\left(B_{2}(t, \cdot)\right)$ with $\phi_{1} \geqq \dot{\psi}_{2}$. Using the technique in 
Lemma 4.1 one sees that (c) in Proposition 4.3 holds. Letting $\bar{\phi}_{0}(\tau) \equiv(1, \cdots, 1) \in$ $\boldsymbol{R}^{M}$ for each $\tau \in \Omega$ and defining $D_{2}(t)=\left\{\phi \in X: \theta \leqq \phi \leqq p(t, \cdot)+\dot{\phi}_{0}\right\}$ for all $t \geqq 0$, one may check that (b) in Proposition 4.3 holds by using (5.7). The fact that (a) holds follows from (5.6) and Lemma 4.2. To see that (d) holds, let $\theta \leqq \phi \leqq$ $p(t, \cdot)+\psi_{0}$ and set $\bar{\psi}=\min \{\phi, p(t, \cdot)\}$. Then $\bar{\psi} \in D_{2}(t)$ and $\bar{\psi} \leqq \dot{\psi}$. Noting that

$$
|\psi-\bar{\psi}|=d\left(p(t, \cdot)-\dot{\psi} ; K\left(\boldsymbol{R}_{+}^{M}\right)\right)
$$

shows that (d) holds (take $N=1$ and $\eta\left(K_{1}, K_{2}\right)$ any positive number in the definition of condition (Q)). Thus Proposition 5.2 is a consequence of Proposition 4.3.

REMARK 5.2. Instead of using the Laplacian operator in this example, one may assume that the operator $L$ has the form $L \phi=\left(\mathcal{A}_{i} \phi_{i}\right)_{1}^{M}$, where each $\mathcal{A}_{i}$ is a second order uniformly elliptic operator in divergence form. Proposition 5.2 remains valid in this circumstance. Proposition 5.1 is also valid if one assumes that either $\Lambda=\prod_{i=1}^{n} J_{i}$ where each $J_{i}$ is a closed interval in $\boldsymbol{R}$ or that $\mathcal{A}_{i}=\mathcal{A}_{1}$ for $i=2, \cdots, M$.

AcKNowledgement. The author is most grateful to the referee for his comments and suggestions.

\section{References}

[1] R.H. Martin, Jr., Approximation and existence of solutions to ordinary differential equations in Banach spaces, Funkcial. Ekvac., 16 (1973), 195-211.

[2] R.H. Martin, Jr., Invariant sets for perturbed semigroups of linear operators, Ann. Mat. Pura Appl., 105 (1975), 221-239.

[3] R.H. Martin, Jr., Invariant sets for evolution systems, International Conf. on Diff. Equations, H. A. Antosiewicz, Ed., Academic Press, New York, 1975.

[4] G.F. Webb, Continuous nonlinear perturbations of linear accretive operators in Banach spaces, J. Functional Analysis., 10 (1972), 191-203.

\section{Robert H. Martin, Jr. \\ Department of Mathematics North Carolina State University Raleigh, North Carolina 27607 U.S.A.}

Added in proof. The idia of using the compactness of $T(t, s)$ in Theorem 3.1 follows from the techniques of Amnon Pazy [Israel J. Math. 20 (1975), 23-36]. 